\title{
POSFET Based Tactile Sensor Arrays
}

\author{
Ravinder S.Dahiya, Maurizio Valle ${ }^{2}$, Giorgio Metta ${ }^{1,2}$, \\ ${ }^{1}$ Italian Institute of Technology, ${ }^{2}$ University of Genova \\ Genova, Italy \\ Ravinder.Dahiya@unige.it
}

\begin{abstract}
In this paper, we present POSFET (Piezoelectric Oxide-FET) based tactile sensing arrays. The equivalent model of a POSFET and its SPICE implementation is presented and various factors involved in the design of POSFET are discussed. A $5 \times 5$ array of POSFET tactile elements designed on the basis of discussion is also presented.
\end{abstract}

\section{INTRODUCTION}

A novel tactile sensing array that comprises of "smart materials" like piezoelectric polymers and the Integrated Circuits is presented. These arrays of tactile sensors are intended to be placed on the distal phalange of the humanoid robot in our lab. Each tactile element on the array comprises of piezoelectric polymer, PVDF-TrFE, directly deposited on the gate area of a FET device. This direct coupling of the transducer to the gate of FET devices significantly reduces the electrical interference and thus improves the signal to noise ratio. Fig. 1 shows the arrangement of a POSFET tactile element. Alternative methods used to develop tactile sensors for robotic applications have been discussed in [1].

A piezoelectric film working in the sensing mode generates a charge/voltage when a mechanical force is applied. This charge/voltage is proportional to the applied stress. By depositing the piezoelectric polymer on the gate area of MOSFET, such a charge/voltage of the piezoelectric polymer can be used to modulate the charge in the induced channel of MOSFET which is then amplified by the MOSFET and further processed by electronic circuitry.

A similar approach was used by Swartz et al. [2] and Fiorillo et al [3] to develop ultrasonic sensor. While the former used the epoxy-adhered PVDF film, later one used a thin film of PVDF-TrFE directly deposited from solution on to the extended gates. In both cases, the extended gate approach was used. In our earlier design, elements of a 32 element microelectrode array test chip were used as extended gates and were epoxy-adhered with $50 \mu \mathrm{m}$ and $100 \mu \mathrm{m}$ films [4]. It was observed that both epoxy and extended gate introduce additional capacitances and hence reduce the sensitivity.

In the following sections, we present the equivalent model of POSFET, its SPICE implementation, design issues and the future direction.

The work presented in this paper has been in part supported by the ROBOTCUB project (IST-2004-004370), funded by the European Commission through the Unit E5 -Cognitive Systems.

\author{
Leandro Lorenzelli \\ Fondazione Bruno Kesslar -IRST \\ Trento, Italy
}

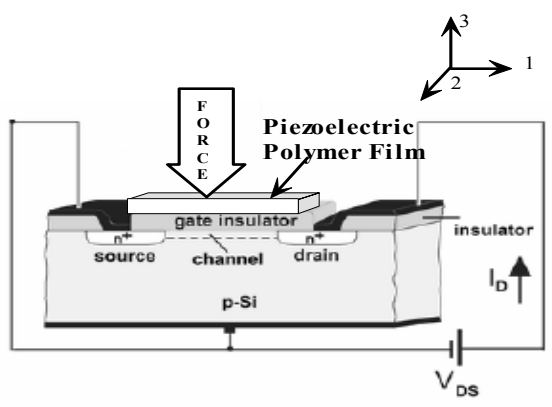

Figure 1. POSFET tactile sensing element arrangement.

\section{Modeling AND ANAlysis}

The electromechanical behavior of piezoelectric polymers is given by following mathematical relations [5]:

$$
\begin{aligned}
& T_{Z}=c_{Z Z}^{D} S_{Z}-h_{Z Z} D_{Z} \\
& E_{Z}=-h_{Z Z} S_{Z}+\beta_{Z Z}^{S} D_{Z}=-h_{Z Z} S_{Z}+\frac{D_{Z}}{\varepsilon}
\end{aligned}
$$

Where $T_{Z}, S_{Z}, D_{Z}, E_{Z}, c_{Z Z}^{D}, h_{Z Z}$ and $\varepsilon$ are the stress, strain, electric displacement, electric field, elastic constant, piezoelectric constant and permittivity respectively. The subscripts correspond to the direction of polarization of polymer and the direction of applied force as shown in Fig 2.

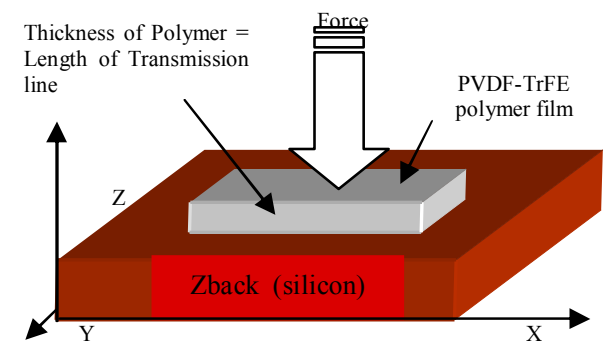

Figure 2. Schmetic of a tactile element

Starting from (1) and (2), a complete model of the piezoelectric polymer (in actuating mode i.e. electrical input 
and mechanical output) including all losses was implemented in SPICE, and is reported elsewhere [6]. In this work, the polymer is used in the sensing mode i.e. mechanical input and electrical output. The SPICE implementation of the equivalent circuit of a POSFET tactile sensing element is obtained by combining the models of piezoelectric polymer (in sensing mode) and MOS device. As shown in Fig. 3, the polymer output is directly coupled to the gate of the MOS device. The transmission line is the electrical analogue of acoustic transmission in polymers. The mechanical losses due to the viscoelastic property of piezoelectric polymers can be considered by using a lossy transmission line. The mechanical to electrical conversion is represented by a transformer which has been implemented with the controlled sources. The gain of controlled sources depends on the piezoelectric constant; hence the piezoelectric losses can be considered by using complex piezoelectric constant. In the electrical block, $C_{\text {polymer }}$ is a lossy capacitor. The dielectric loss in the polymer has been implemented by implementing lossy $C_{\text {polymer }}$ and is shown in the Fig. 3. The simulated output of the POSFET model, obtained by applying a $0.1 \mathrm{~N}$ step force at one end of the transmission line is shown in Fig. 4. The other end of transmission line is connected with the electrical analogue of mechanical impedance of silicon. Level II model of MOS devices was used during simulation. The model can be used over a wide range of frequencies ( $\sim \mathrm{MHz})$.

The implementation of polymer + FET model in SPICE is advantageous as it enables sensor optimization based on both, polymer parameters and those of conditioning electronics.

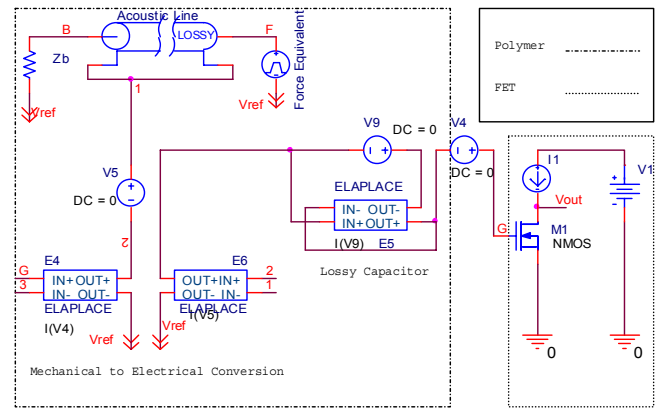

Figure 3. Equivalent model of POSFET tactile element implemented in PSpice.

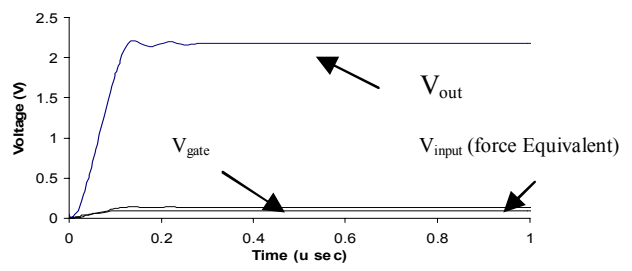

Figure 4. Simulated output of the POSFET tactile element, at drain terminal (top), gate termimal (middle) and input (below) $A=0.196 \times 10^{-6} \mathrm{~m}^{2}$, $Z=50 \mu \mathrm{m}, \varepsilon=70 \times 10^{-12}, h_{33}=4.4 \times 10^{9}$ are used is all simulations.

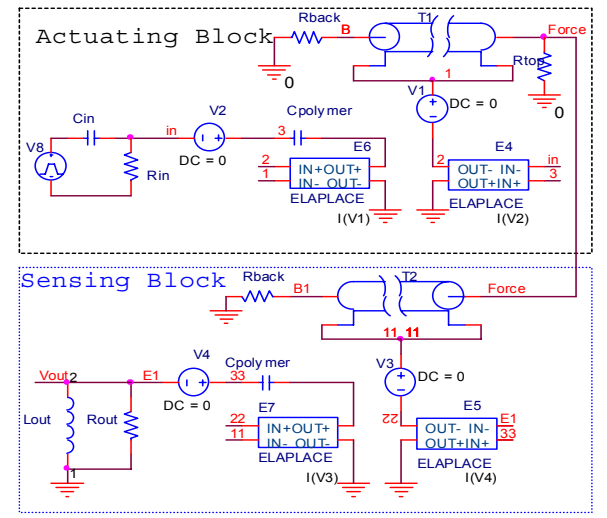

Figure 5. The arrangement to verify the SPICE model of polymer.

Since it is difficult to apply forces over wide range of frequencies $(\sim \mathrm{MHz})$ by a mechanical arrangement, the polymer model in sensing mode was evaluated by using the SPICE model of the polymer in actuating mode to produce the force which is then fed in to the SPICE model of polymer (sensing mode). This arrangement, as shown in Fig 5, is similar to pulse-echo method which is explained in [5]. The performance of polymer model in sensing mode was evaluated by comparing the simulated response with experiment results reported in [7]. For comparison, the electrical input impulse to the transmitting stage, the load at the output terminal of sensing stage, and various constants were kept same as that used in [7]. The input to the actuating stage is a voltage impulse ( $300 \mathrm{~V}$, fall time $100 \mathrm{~ns})$ which is generated by $\mathrm{R}_{\mathrm{in}}=100 \Omega$ and $\mathrm{C}_{\mathrm{in}}=2 \mathrm{nF}$. The output of the sensing stage is terminated in to a load comprising a resistance $\left(\mathrm{R}_{\text {out }}=100 \Omega\right)$ and inductance $\left(\mathrm{L}_{\text {out }}=4.7 \mu \mathrm{H}\right)$ connected in parallel. The losses could not be considered in this comparison because the constants used in [7] are real numbers. Fig. 6 shows that the simulated output of the polymer in sensing mode is in good agreement with the experiment results and hence the model in Fig. 3 can be used for design and analysis of POSFET tactile sensor elements.

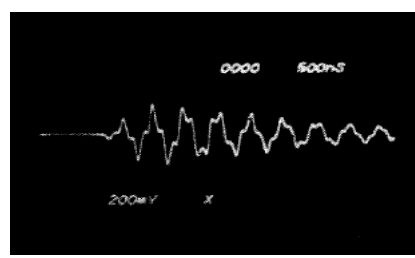

(a)

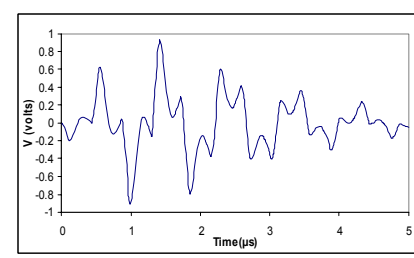

(b)
Figure 6. (a) Experiment result reported in [6], (b) Simulated response of SPICE model of piezoelectric polymer in sensing mode.

\section{DiscusSION}

The model of POSFET tactile element shown in Fig. 3 can be used to get the insight in to the design of tactile sensing array under different conditions. The performance of the sensors depends on both the polymer film and the design of FET devices. The factors related to polymer and FET device are discussed below: 


\section{A. Factors related to polymer:}

Various factors that influence the response of polymer are its thickness, material on front and back of the polymer, area of the polymer film, and electrodes [8]. The open circuit voltage of a piezoelectric polymer, when a step force is applied on top, as shown in Fig. 2 is:

$$
\begin{aligned}
& V_{\text {polymer }}=-\frac{h_{z z} \times F_{i n} \times\left(1-R_{f p}\right)}{Z_{a p}} \times\left[t-\left(1+R_{p b}\right) \times(t-(Z / v)) \times\right. \\
& u(t-(Z / v))+R_{p b} \times\left(1+R_{f p}\right) \times(t-(2 Z / v)) \times u(t-(2 Z / v))- \\
& \left.R_{f p} \times R_{p b} \times\left(1+R_{p b}\right) \times(t-(3 Z / v)) \times u(t-(3 Z / v))+\ldots\right]
\end{aligned}
$$

Where, $\quad R_{f p}=\left(Z_{p}-Z_{f}\right) /\left(Z_{p}+Z_{f}\right)$ represent the reflection coefficient at front-polymer interface and $R_{p b}=\left(Z_{p}-Z_{b}\right) /\left(Z_{p}+Z_{b}\right)$ at polymer-back faces. $Z_{p}, Z_{f}$, and $Z_{b}$ are the acoustic impedances of polymer, and the material on the front and back (silicon in our case) sides respectively. $Z$ is the thickness of the polymer, $v$ is the wave velocity in polymer and $u($.) is the step function. It can be noted from (3) that the open circuit voltage of the polymer depends on the thickness of the polymer, the reflection coefficients at the two faces, internal parameters like piezoelectric constant and elastic constant (acoustic impedance). In addition to these, the capacitance of polymer is also involved when the polymer is connected with a load.

The internal properties like piezoelectric constant depend on the way the polymer is made. A uniform thin film of PVDF-TrFE can be obtained by spin coating the polymer solution and annealing it at around $150^{\circ} \mathrm{C}$ and reducing the temperature slowly [3]. In order to have piezoelectric properties, the piezoelectric polymer needs to be poled, which requires application of high voltage $(\sim 50 \mathrm{~V} / \mu \mathrm{m})$. Extreme care is required in case of POSFET, as the poling is done in situ, and high voltage may change the device properties itself. A polymer film with 5-10 $\mu$ m thickness can be poled without damaging the device.

The materials on the front and back of the polymer offer different acoustic impedances to the force waves. The response of polymer with different materials under polymer simulated with the SPICE model presented earlier is shown in Fig. 7. It can be noticed that the polymer has higher sensitivity when a stiff material is used on the back side. For stiffer materials, the reflection coefficient $R_{p b}$ is high and the device operates in $\lambda / 4$ mode. In this work, silicon is present on the back side and it has the acoustic impedance approximately five times that of PVDF-TrFE. Thus, the reflection coefficient ' $R_{p b}$ ' is 0.66 . Hence the presence of silicon under the polymer ensures a higher sensitivity.

In (3), the term outside brackets represents the net mechanical to electrical conversion and the terms inside the bracket are combination of incident and reflected force waves. It can be observed from the first term inside brackets that the voltage is a ramp function. If the thickness of the

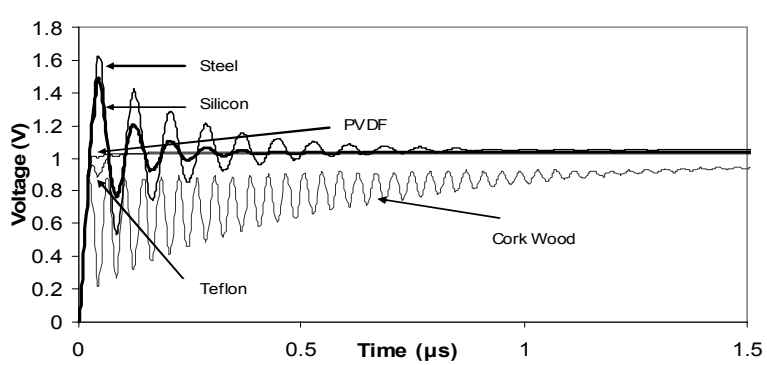

Figure 7. Response of polymer with different backside materials. Acoustice impedance of steel, silicon, PVDF, teflon , \& wood are 9, 3.8, 0.8, 0.6 and 0.02 MRayl respectiely. A step force of $0.01 \mathrm{~N}$ was applied.

polymer is increased, the step function terms are further delayed (delay time increases as $\mathrm{Z}$ is increased). Thus the open circuit voltage keeps on increasing linearly until first delay term contributes to the net voltage. Thus, the open circuit voltage in general increases with the thickness of the polymer film. This thickness effect of polymer film can also be noted from the simulation result shown in Fig. 8. Apart from above discussed factors, the response of polymer also depends on its area, which is related with the FET design.

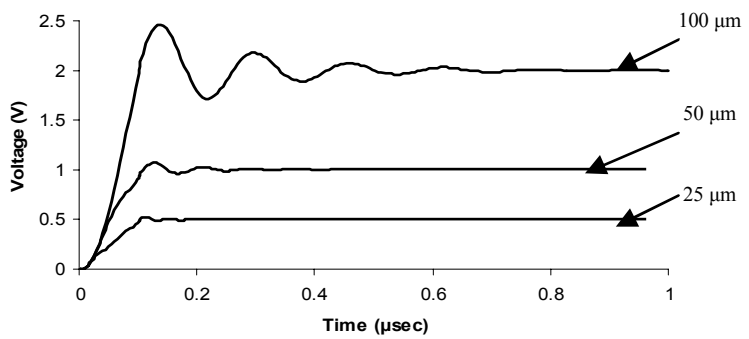

Figure 8. Simulated open circuit voltage of 25,50 and $100 \mu \mathrm{m}$ thick polymer film. A step force of $0.1 \mathrm{~N}$ was applied.

\section{B. Factors related to FET Device:}

The small signal model of POSFET tactile element in common source arrangement is obtained from Fig. 1 and 3 and is given in Fig. 9. For simplicity, the source and body are assumed to be connected to ground. The open circuit of voltage of polymer $V_{\text {polymer }}$ transferred to gate terminal of FET is:

$\frac{V_{g}}{V_{\text {polymer }}}=\frac{C_{\text {polymer }}}{C_{\text {polymer }}+C_{g s}+C_{\text {sub }}+C_{g d}\left(1+A_{v}\right)}$

Where, $A_{v}$ is the voltage amplification of the transistor. It can be noticed from (4) that for higher voltage transfer from polymer to gate terminal, the capacitances of FET device should be low with respect to that of polymer. Other parasitic capacitances have been ignored because polymer is directly coupled to FET device. In this work, $C_{\text {sub }}$ is zero as the polymer coupled to the gate area $\left(C_{\text {sub }} \neq 0\right.$, if extended gate is used). The net capacitance seen by polymer can be reduced 


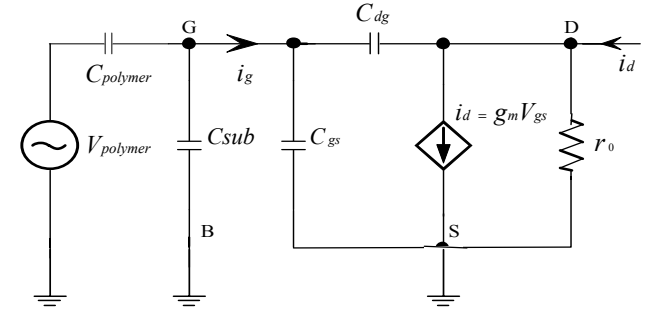

Figure 9. Small signal model of POSFET. $C_{\text {sub }}$ is the substrate capacitace due to extended gate (shown only to indicate the effect of substrate, in our present case this capacitance is not present).

by designing a transistor with smaller $\mathrm{W} / \mathrm{L}$. But, in order to have the larger voltage gain and to reduce the noise, a higher $\mathrm{W} / \mathrm{L}$ ratio is desired. The effect of the input capacitance of the transistor can be reduced by using the source follower arrangement, but this will increase the noise and non linearity [9].

It can be noticed from (4) that the voltage transferred to the gate terminal, also depends on the capacitance of the polymer. Higher voltage ratio can be obtained by increasing the capacitance of the polymer with respect to the input capacitance of the FET device. This means reduction in the thickness of polymer, if the area $(=W \times L)$ is assumed constant. But as argued earlier, reduction in thickness will reduce $V_{\text {polymer }}$ and hence a trade-off is required in the design. Substituting $C_{\text {polymer }}=\varepsilon_{33} A / Z$ in (4), the thickness of polymer required to transfer more than eighty percent of the asymptotic $(t=\infty)$ value of $V_{\text {polymer }}$ to the gate terminal is given by [3],

$$
Z \geq 4 \varepsilon_{33} A /\left(C_{g s}+C_{g d}\left(1+A_{v}\right)\right.
$$

The substrate capacitance $C_{\text {sub }}$ is not included in this expression. For example, if $W=1200 \mu \mathrm{m}, L=4 \mu \mathrm{m}, C_{g s}$ $+C_{g d}\left(1+A_{v}\right)=2 \mathrm{pF}$, and $\varepsilon_{33}=4$, at least $85 \mathrm{~nm}$ thick polymer is required for eighty percent voltage transfer.

\section{Device Design}

Test arrays with $25 \mathrm{n}-\mathrm{MOS}$ elements are developed at FBK-IRST and a prototype is shown in Fig. 10. In order to have large tranconductance, the $\mathrm{W} / \mathrm{L}$ ratio was made 300 . A $\mathrm{Si}_{3} \mathrm{~N}_{4} / \mathrm{SiO}_{2}$ double layer is used as a gate dielectric. The reference fabrication process for this design is the n-MOS technological module of a non standard $4-\mu \mathrm{m} \mathrm{Al}$ gate p-well ISFET/CMOS technology [10]. In order to get a working mode "POSFET", PVDF-TrFE will be spun onto the whole wafer surface. Due to the requirement of in situ poling of polymer, the thickness of polymer would be restricted to $10 \mu \mathrm{m}$. For poling, a metal layer is required between the polymer and gate oxide. During poling, the metal on gate, the source, drain, and body will be kept at same potential to avoid the device damage. Piezoelectric polymers are sensitive to temperature variations also. A temperature

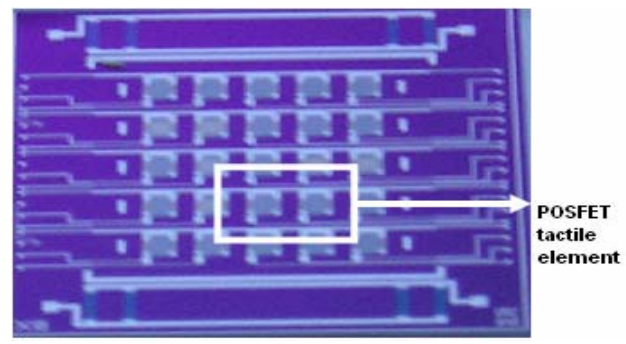

Figure 10. Array prototype $(1 \mathrm{~cm} \times 1 \mathrm{~cm}$ die size $)$.

sensing device is made available on chip to reduce the temperature variation.

\section{CONCLUSION AND Future Work}

The equivalent model of POSFET and its SPICE implementation has been presented. The simulated response of the model is in agreement with the experimental results. Various issues involved in the design of POSFET tactile element have been discussed theoretically and by simulation. The working mode of POSFET will be obtained by spin coating the polymer on the FET array. The poling of the polymer in situ would be challenging as it requires application of high voltage. The devices will be further tested after depositing the polymer film on the array of FETs. In future the tactile sensing system i.e. POSFET tactile sensing arrays and the conditioning electronics and other functional blocks will be integrated on the same chip. This integration will reduce the number of wires and hence; will also address the problem of wiring complexity in robots.

\section{REFERENCES}

[1] M.H. Lee and H.R. Nicholls "Tactile sensing for mechatronics - a state of art survey", Mechatronics, 9(1), October 1999.

[2] Robert G. Swartz, James D. Plummer, "Integrated silicon-PVF2 acoustic transducer arrays," IEEE transactions on Electron Devices, Vol. 26, No-12, Dec 1979, pp 1920-32.

[3] A.S. Fiorillo, J. Van Der Spiegel, P.E. Bloomfield and D. EsmailZandi, " A P(VDF-TrFE) based Integrated Ultrsonic Transducer," Sensors and Actuators, A21-A23, 1990,pp. 719 - 725.

[4] Ravinder S. Dahiya, M. Valle, G. Metta, L. Lorenzelli Cristian Collini, "Tactile sensing arrays for humanoid robot," IEEEPRIME'07, The 3rd Conf. on PhD research on Microelectronics and Electronics, Bordeaux, France, pp 210-204, July 2007.

[5] ANSI/IEEE Standard of Piezoelectricity, 176-1987.

[6] Ravinder S. Dahiya, M. Valle, G. Metta, L. Lorenzelli, "Modeling of Piezoelectric Polymers in SPICE," 2007 MRS Fall meeting (unpublished).

[7] G. Hayward, M.N. Jackson, "Discrete-time modeling of the thickness mode piezoelectric transducer", IEEE Trans.Sonic \& Ultrason., vol SU-31, no 3, May 1984, pp 137-66.

[8] Lewis F. Brown, "Design Considerations for Piezoelectric Polymer Ultrasound Transducers," IEEE Trans. Ultrasonics, Ferroelectrics and Freq. Control, Vol. 47, no. 6, Nov 2000, pp. 1377-1396.

[9] Behzad Razavi, "Design of Analog CMOS Integrated Circuits," MacGraw Hill, 2000.

[10] S. Martinoia, et. al, "Development of ISFET array-based Microsystems for bioelectrochemical measurements of cell populations," Biosensors \& Bioelectronics, 16, 2001, pp 1043-1050. 